\title{
Visibility of an afterimage alone and in the presence of one or two additional afterimages*
}

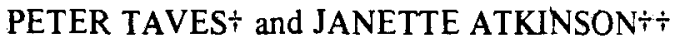 \\ The Johms Hopkins University, Baltimore, Maryland 21218
}

\begin{abstract}
The total time for which a circular afterimage is visible has been found to be increased by the presence of one or two additional afterimages. This increased visibility, which has been called "facilitation," is greater the nearer the afterimages are to one another, being optimum at .25 deg and declining to zero at 2.0 deg separation.
\end{abstract}

In a study of fragmentation of partially stabilized images, Pritchard, Heron, and Hebb (1960) discovered that a figure occupying one part of the stimulus field could affect the visibility of a nearby figure in the same stimulus field. These authors considered this phenomenon to be a Gestalt "field effect," similar to the effects found by Köhler for real images (1940. 1944).

More recently, Atkinson $(1970,1972)$ has conducted extensive research to determine how the presence of one afterimage affects the total unitary visibility of another. A series of patterns of pairs of rectangular afterimages in varying spatial relationships to each other was used. Both proximity and relative orientation were found to affect the visibility of each afterimage: a bar's visibility was increased by the presence of a second bar in the same orientation; visibility of two similarly oriented bars was augmented as their proximity increased, with maximum visibility occurring when the bars were $.50 \mathrm{deg}$ apart .

The present two experiments were conducted to determine the total unitary visibility of a single circular afterimage, both alone and in the presence of either one or two additional circular afterimages in specified spatial relationships to the first. In the present two experiments, the afterimages were of circular disks with no orientation properties. The aim of these experiments was to ascertain whether field effects could be found independent of orientation interactions and. if found, to measure the factors limiting the effect.

\footnotetext{
*This research was supported in part by a contract between the Engineering Research Program. Office of Naval Research. and the Johns Hopkins Lniversity. The authors wish to thank Dr. Howard Egeth and Dr. William Bevan for their encouragement and advice on methodological problems.

$\leftarrow$ Now at the Department of Psychology. Princeton Lniversity. Princeton. New Jersey 08540.

$\div$ Now at the Physiological Laboratory, the Lniversity of Cambridge. Cambridge. England.
}

\section{EXPERIMENT I}

Method

Apparatus

All afterimages were produced by an Ultrablitz Cornet 150 electronic flash unit (light output $=1,250 \mathrm{BCPS}$; flash duration = $.625 \mathrm{msec}$; color temperature $=5,000^{\circ} \mathrm{K}$, corresponding to medium daylight). A diffusion filter was placed in front of the flash unit. The flash was given in a dimly illuminated room. Patterns for producing the afterimages were constructed by cutting holes in a black mask. The patterns were placed directly in front of the diffusion filter for viewing. The apparatus was constructed so that no stray light escaped when the flash was triggered.

\section{Subjects}

Sixteen student volunteers from The Johns Hopkins University served as naive $S s$ in Experiment 1. All had normal or corrected vision; none had any color vision defects as ascertained by H-R-R pseudoisochromatic plates.

\section{Procedure}

Afterimages were viewed binocularly. The $\mathrm{S}$ fixated a small white dot painted on the mask. His eyes were always $28.5 \mathrm{in}$. from the face of the patterns and were vertically and horizontally aligned with the fixation point. Immediately after the flash, the $\mathbf{S}$ closed his eyes and kept them closed for the duration of the trial. thus maintaining a constant background luminance for observing the afterimage.

Each trial lasted $60 \mathrm{sec}$, commencing when $S$ first depressed a microswitch to signal initial complete visibility of the afterimage. The $\mathrm{S}$ was instructed to keep the microswitch depressed whenever the afterimage was totally visible and to release the switch whenever the image was not entirely visible. No results were disclosed during the experiment.

The $S$ was given a 10 -min period for dark adaptation prior to the first trial. and a 5 -min recovery period between trials. Only the initial minute of the afterimage's duration was used in the analysis. because afterimages typically became faint and difficult to recognize after a minute. The $S$ was instructed to make as few voluntary eve movements as possible. He was cautioned not to blink. rub his eyes. or squint during the trials.

Total unitary visibility was measured in seconds of trial time for which the response button was depressed, as indicated on the clock attached to the microswitch. The $S$ was given one practice trial on each of three patterns: a one-disk. a two-disk, and a three-disk pattern selected randomly from among the set of five 


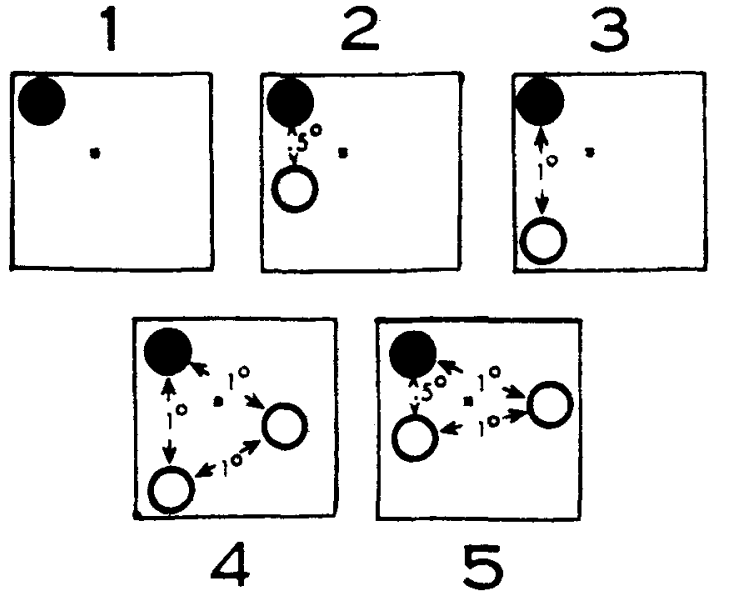

Fig. 1. Pattems used in Experiment 1. Black disks represent target disk cutouts: rings represent nontarget cutouts. All disks are actually identical (round. $.50 \mathrm{deg}$ in diam). Small black squares represent fixation points. All distances shown represent edge-to-edge measurements.

Table 1

Total Unitary Visibility (Seconds)

\begin{tabular}{ccc}
\hline Pattern & Mean & SD \\
\hline 1 & 33.82 & 10.49 \\
2 & 43.54 & 10.51 \\
3 & 38.22 & 0.84 \\
4 & 41.48 & 9.59 \\
5 & 42.16 & 9.63 \\
\hline
\end{tabular}

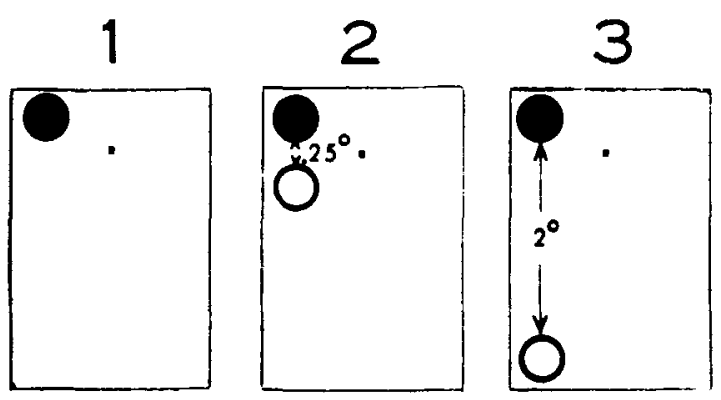

Fig. 2. Patterns used in Experiment II. (See Fig. 1 for detailed explanation.)

patterns used in the experimental trials. Between and after the three practice trials. $S$ was allowed to ask questions about procedure. The five patterns used in the eyperimental trials were presented in random order. with two trials for each pattern.

\section{Patterns}

The five patterns used in Experiment I are represented in Fig. 1. For each pattern. $S$ was instructed to respond to the visibilits of only one afterimage (the "target disk"). The target disk was always located in the same position relative to the fixation point and .50 deg away from it.

\section{Results}

Mean visibility scores corresponding to the patterns represented in Fis. I dre given in Table l. A two-wa! and sis of bariance indicated a significant difference berween patterns. $F(t .60)=1+.65 . p<.01$. To andilye the results in more detail. a post hoc series of $t$ tests was condusted. applying the Scheffe correction for nonindependent comparisons (Ferguson. 1966. $p F,(-15-2,7)$ ). The difference between mean visibility for Patterns 1 and 2 was significant. $t(15)=6.11 . p<.01$. as Was that for Patterns 1 and 3. $t(15)=4.02 . p<.01$, and for Parterns 2 and 3. $t(15)=4.63 . p<.01$. All three of these results support the findings of Atkinson in that facilitation was found to decrease with separation of similarly oriented bars. Patterns 4 and 5 were each significantly higher than Pattern 1 in total unitary visibility (in both comparisons $\mathrm{p}<.01$ ). while Patterns 4 and 5 were not significantly different from each other or from Patterns 2 or 3.

\section{Discussion}

This last finding was initially somewhat puzzling. The expectation was that if Patterns 2 and 3 were significantly different from each other. then perhaps Patterns 4 and 5 would also be significantly different from each other. since only another disk had been added to each of Patterns 2 and 3 to get Patterns 4 and 5 . One plausible explanation of this result is that in Pattern 5. the $.50-\mathrm{deg}$ separation may provide maximum facilitation of visibility of the target disk. so that the presence of the third disk cannot increase facilitation any further (hence there is no significant difference between Patterns 2 and 5 in terms of mean visibility). In contrast. facilitation in Pattern 3 is not at a maximum (because proximity of the two disks is not great enough). so that in Pattern 4. the presence of the third disk may contribute some extra facilitation. making facilitation in Pattern 4 somewhat higher than in Pattern 3.

\section{EXPERIMENT II}

Experiment II was carried out to measure the extent of facilitation for larger and smaller separations than those used in Experiment I. The apparatus and procedure were the same as in Experiment I. Eight new naive Ss were used. Three patterns were used. as shown in Fig. : .

\section{Results}

The two-dish pattern with .25-deg separation (Pattern 2. Fig. 2) had the highest visibility score (mean $=42.37 \mathrm{sec} . \mathrm{SD}=10.84 \mathrm{sec})$ : the two disk patten with 2.0-deg separation (Pattern 3. Fig. 2) had the second highest mean visibility score $($ mean $=33.55$ sec. $\mathrm{SD}=$ 
Fig. 3. Facilitation as a function of separation between disks.

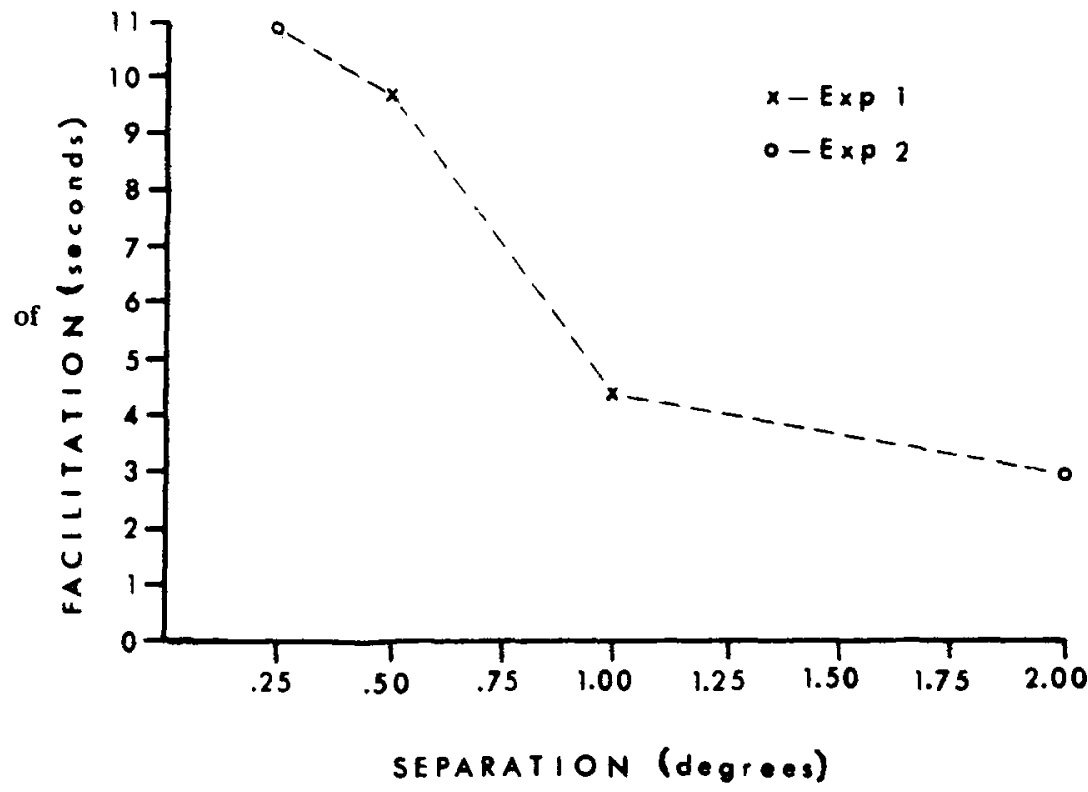

$12.66 \mathrm{sec}$ ): the single disk (Pattern 1. Fig. 2) was lowest in terms of total unitary visibility (mean $=30.57 \mathrm{sec}$. SD $=13.44 \mathrm{sec}$ ). A two-way analysis of variance yielded a significant $F$ value for pattern differences, $F(2,14)=$ 12.31. $\mathrm{p}<.01$.

The visibility of Pattern 2 was significantly greater than that of the other two patterns. with $t(7)=5.33$. $p<.01$ for Pattern 1 vs Pattern 2 and $t(7)=4.23$, $p<.01$ for Pattern 2 vs Pattern 3. However, total unitary visibility for Pattern 3 (2.0-deg separation) was not significantly greater than that for Pattern 1 (single disk), $t(7)=1.61, .20>p>.10$. These findings show that facilitation is highest for two disks that are close together $(.25 \mathrm{deg})$ and does not exist for disks which are 2.0 deg apart

\section{DiSCUSSION}

Taken together, the results from Experiments I and II lend strong support to the conclusion. indicated by the previous results of Atkinson (1970, 1972), that the presence and proximity of a second stimulus in the visual field can facilitate visibility of the primary stimulus. The results indicate that facilitation does not depend on the orientation of images. for a strong effect has been found for images with no orientational properties. If the results from Experiments I and II are combined (Fig. 3). there is a gradual decrease in facilitation with increase in separation. with maximum facilitation effect at approximately .25 deg. However. these results come from different groups of Ss. and conclusions about combined findings can be only tentative. If the distance separating the disks is less than $.25 \mathrm{deg}$."losure" of the two afterimages often occurs and it becomes impossible to clearly identify the disks. For this reason. no separations smaller than .25 deg were used in these experiments.

It seems likely that the observed facilitation represents a perceptual enhancement process and depends upon an interaction of functional groups of receptors at some level of the visual system. The effect may be related to "preattentive processes" as put forward by Neisser (1967). These processes are prerequisite to any perceptual analysis: they represent preliminary visual enhancement at appropriate places in the receptive field. in preparation for more discriminative analyses. There is little strong evidence as to where in the visual system such processes might occur, although previous research using dichoptic viewing has shown that the facilitatory interactions are beyond the level of the visual system at which binocular combination of information occurs (Atkinson, 1972). One possible site is the superior colliculus, for lesion studies by Schneider (1969) suggested that some collicular units are involved mainly in processes of simple visual enhancement rather than detailed stimulus analysis. However, on the basis of the present results. no definite postulations can be given concerning the exact properties of the units involved. the actual physical nature of their interaction, or their precise location in the visual sy'stem.

\section{REFERENCES}

Atkinson. 1. A study of perceptual analysis using stabilized images. Lnpublished PhD thesis. Iniversity of Cambridge. 1970 .

Atkinson. J. Visibility of an afterimage in the presence of a second afterimage. Perception \& Psychophysics. 1972.12. $257-262$.

Perguson. G. A. Statistical analysis in psychologr and education. Vew York: MoGraw-Hill. 1966.

Kuhler. W. Dinamics in psichology. Aew York: Liveright. 1940. Kohler. W. Iigural aftereffecte: An mestigation of bisul 
processes. Procedings of the American Philosophical Sociery. 1944. 88. 269-357.

Deiser. L. Cognitire pishologr. Dew lork: Appleton-Centurydrofts 196?

Pritcharu. R. It. Heron. II.. \& Holbb. D. O. Visual perception approashed by the method of stabilized images. Canadian lournal of Psichology. 1960. 2.67-77.
Schneider. G. E. Two risual systems-hrain mechanisms for localization and discrimination are associated by tectal and cortical lesions. Science. 1969. 163. 895-902.

(Received for publication June 19. 1972: revision received Jul! 26. 1972.1 